\title{
CORRECTION TO: INTEGRABILITY OF CLASSICAL AFFINE $W$-ALGEBRAS
}

ALBERTO DE SOLE

Dipartimento di Matematica Sapienza Università di Roma P.le Aldo Moro 2, 00185 Rome, Italy desole@mat.uniroma1.it

VICTOR G. KAC

Department of Mathematics MIT

77 Massachusetts Avenue Cambridge, MA 02139, USA kac@math.mit.edu
MAMUKA JIBLADZE

\author{
School of \\ Mathematics and Statistics \\ University of Glasgow \\ G12 8QQ Glasgow, UK \\ daniele.valeri@glasgow.ac.uk
}

Correction to: Transformation Groups 2021, 26(2): pp. 479-500

https://doi.org/10.1007/s00031-021-09645-0

The original version of this article unfortunately contained some mistakes in web displays of author order.

This has been corrected to read Alberto De Sole, Mamuka Jibladze, Victor G. Kac, Daniele Valeri. 